\title{
À PROPOS DE L'ÉVOLUTION DES VOYELLES INITIALES E O.
}

Francisco Javier Deco Prados

Universidad de Cádiz

\section{RÉSUMÉ}

Je me propose ici d'étudier uniquement l'évolution des voyelles initiales e o suivies de consonne orale non palatale, ce que Fouché appelle évolution indépendante de l'articulation des phonèmes voisins, sauf cas d'entrave générique. Je ne traiterai donc pas, par exemple, les modifications dues aux processus de palatalisation ou de nasalisation. Je ne parlerai pas non plus des cas de chute de ces voyelles. Je voudrais insister sur le fait qu'on ne fera pas l'histoire complète de ces voyelles en cette position: le but de cette étude est d'analyser les principales évolutions du e et du o initiaux pour essayer de clarifier les très intéressants problèmes qui, à leur égard, se sont posés aux XVIe et XVIIe siècles.

\section{1. Évolution de la voyelle E.}

Vers le IVe siècle, les voyelles initiales e o ne seront que fermées (v., par exemple, Zink, 1989: 71). Matte précise qu'elles seraient en réalité des sons intermédiaires, en ce sens que la voyelle palatale serait fermée mais peu écartée et la voyelle vélaire fermée aussi mais peu arrondie. Il date ces aboutissements respectivement du IIIe et du IVe siècles et les appelle e, o centralisés. En fait, 
d'après les théories de cet auteur, une période de relâchement général de l'acte de phonation commence en ce moment. Les mâchoires en action pour la fermeture des éléments prévocaliques ont provoqué la diminution du jeu de la langue et des lèvres. Les voyelles se centralisent, deviennent, selon les cas, moins arrondies ou moins écartées, moins palatales ou moins vélaires. (Cf. Matte, 1982: $98-99$ et 107-108).

A partir de ce moment, le e centralisé (ę) en position entravée devant une consonne orale non palatale, se rapproche un peu plus de la voyelle neutre e central (ę), mi-ouverte, s'ouvrant en ę. Les Bourciez (1974: 55 et 107) proposent la date du milieu du XIIe siècle tenant compte du changement analogue des voyelles toniques entravées d'après le témoignage des assonances. Fouché (1969: 430) et Zink (1989: 71) donnent la même date. Matte (1982: 156) considère que le changement se produit probablement plus tôt, à l'époque prélittéraire.

Il faut voir que l'effacement d'un $s$ implosif après la voyelle initiale a empêché la transformation en ę, ce qui vient confirmer la date du changement. Le $s$ sonore disparaît devant consonne sonore pendant la première moitié du XIe siècle. Devant consonne sourde, vers les mêmes dates que le changement du e. Pour le passage, dans ce cas, de ę à ẹ, il faut considérer la période où les modes tendu et croissant l'emportent, l'époque du moyen français qui, pour Matte, s'étend du XIIIe au XVIe siècle (cf. Matte, 1982: 133-134).

En position libre devant consonne orale non palatale, le ę est plus faible. II faut considérer que l'entrave compensait dans le cas antérieur le processus d'affaiblissement. A présent, la voyelle se centralise davantage et arrive à e. Les Bourciez (1974: 107) signalent seulement que le changement est très ancien. Fouché (1969: 430) ne foumit pas non plus de date exacte mais indique l'époque prélittéraire. La Chaussée (1989: 89) et Zink (1989: 71) donnent le XIe siècle, tandis que Matte (1982: 108 et 156) propose aussi, de nouveau, l'époque prélittéraire.

Postérieurement, quand le mode tendu aura repris le dessus, vers le XVe siècle, ce son central va s'antérioriser et se labialiser, se transformant en $\propto$ (voyelle palatale arrondie intermédiaire).

Je dois maintenant parler du mouvement de réaction savante qui part, au XVle siècle, de la réforme érasmienne de la prononciation latine et qui changera la prononciation $\propto$ en ẹ en position initiale dans un grand nombre de mots. Son influence sur la voyelle o sera analysée plus tard.

Erasme, opposé à l'esprit parfois trop conformiste de certains humanistes italiens, entreprend pendant les années 20 du XVIe siècle la rédaction du $\mathrm{Ci}$ ceronianus, traité qui, d'après Renaudet «allait définir l'éthique de son humanisme» (Renaudet, 1956: 190), fondé sur le refus des conventions sociales et littéraires et sur le souci de parvenir à une expression exacte et sincère. En mê- 
me temps, Erasme va écrire son De recta latini graecique sermonis pronunciatione, dédié en février 1528 à Maximilien de Bourgogne et publié en un volume avec le Ciceronianus en mars de la même année. On pourrait dire que ce dialogue était une sorte de preuve de finesse et de connaissance relative aux deux langues les plus prestigieuses du moment. Erasme voulait en fait restituer la vraie prononciation du latin et du grec à l'époque de leur apogée, et pour y parvenir il a étudié la pratique des transcriptions du grec au latin, l'orthographe archaïque du latin, les données de la métrique et de la prosodie, les témoignages des grammairiens antiques et médiévaux et il a aussi utilisé la comparaison avec les langues modemes. (Cf. Renaudet, 1956: 191).

La prononciation du grec transmise par les Byzantins était très modifiée mais la dégénérescence de celle du latin était encore plus grave. Chaque peuple d'Europe prononçait à sa façon. Erasme raconte qu'à l'occasion d'une réception de l'empereur Maximilien, un orateur français avait lu un texte en latin et que les Italiens présents avaient cru qu'il parlait en français. Il en arrive à affirmer que c'est en France qu'on prononce le plus mal le latin. (Cf. Renaudet, 1956: 191-192).

Mais Erasme n'était pas le seul à critiquer la prononciation française du latin. Tory fut l'un des premiers partisans de sa réforme orthographique et phonétique, mais, comme Beaulieux l'indique dans son Histoire de l'orthographe française (1967:222), il partait essentiellement du modèle italien de prononciation. Erasme avait inauguré en ce sens l'étude de la phonétique chez les grammairiens de l'Antiquité et son De recta fut réimprimé plusieurs fois en France, en $1528,1530,1547$, etc.

D'autre part, Charles Estienne écrit, pour l'instruction de son neveu et de Baif, un ouvrage analogue: De recta Latini sermonis pronunciatione et scriptura, qui sera édité en 1538 et en 1541 . Beaulieux signale que, grâce à l'Italie et aux livres d'Erasme et de Charles Estienne, «la réforme de la prononciation latine était déjà, au milieu du siècle, bien avancée» (Beaulieux, 1967:225). Les humanistes, surtout à partir de cette date, vont réagir contre la prononciation barbare du latin et le mouvement de restitution prendra une telle ampleur qu'il va dépasser le cadre du latin pour influencer la prononciation du français. Beaulieux affirme qu' «à mesure que grossit le nombre d'humanistes qui s'intéressent à la langue vulgaire (...), l'évolution de la prononciation des mots de la langue parlée subit une entrave de plus en plus forte, tandis qu'il y a des changements importants dans la prononciation des mots de la langue écrite (...). Brunot dit avec très juste raison qu'il n'est plus question maintenant d'évolution phonétique mais de modification de la prononciation» (Beaulieux, 1967:232).

A la fin du Moyen Age on prononçait le e initial dans les mots latins avec un $\propto$ comme en français. La restitution commence donc par le latin et gagne la 
langue française à partir du milieu du XVIe siècle et pendant le XVIIe siècle, se faisant sur la prononciation ẹ.

Thurot, dans De la prononciation française depuis le commencement du $X V l e$ siècle d'après les témoignages des grammairiens, ouvrage de base qui malgré la date de parution, 1881, reste fondamental, affirme que le changement de $\propto$ en e dans les voyelles atones initiales s'est produit dans un assez grand nombre de mots surtout depuis la fin du XVIe siècle (Cf. Thurot, 1966, I: 121 122 et 129 ).

Il part de la base (cf. Thurot, 1966, I: 108) que dans presque tous les mots savants, c'est-à-dire ceux qui ne sont pas passés par l'usage populaire, le e atone est fermé, notamment dans de nombreux mots commençant par les préfixes dé, ré, pré. Ailleurs, le passage du e féminin (œ) à ẹ, se produit, comme on vient de le dire, très fréquemment.

Ferdinand Brunot, dans le volume consacré au XVIe siècle (cf. Brunot, 1967: 244), se limite à donner une liste des mots où l'on observe le changement, alléguant une influence latine ou dialectale. Dans le tome IV de son Histoire (Brunot, 1966b: 193-195) il traite le problème de la transformation de e initial au XVIIe siècle. De façon surprenante il reproduit les pages que Théodore Rosset avait consacrées au problème dans son travail de 1911 Les origines de la prononciation moderne étudiées au XVIle siècle d'après les remarques des grammairiens et les textes en patois de la banlieue parisienne, ouvrage de base aussi, qui est encore fondamental pour l'étude de la prononciation de l'époque.

Rosset (1911: 145-152), donc (et Brunot qui accepte ses thèses), nie la possibilité d'un processus phonétique de délabialisation de $\propto$ en position initiale. Il part de l'observation des listes fournies par Thurot pour affirmer que le passage de $\propto$ à ẹ au cours du XVIe et du XVIIe siècle est dû essentiellement à l'action combinée de l'influence savante et de l'analogie. Il systématise les principaux changements - les plus nombreux - en quatre groupes: 1) Dans les formes atones des verbes sous l'influence du radical tonique (téter, péter, etc.). 2) Dans les mots dérivés sous l'influence du mot primitif (lévrier, chérir, etc.). 3) Sous l'influence des préfixes savants dé et ré (défendre, débuter, réduire, etc.). 4) Par la similitude dans l'écriture du mot latin et du mot français (péril, séduire, désir, etc.).

Fouché (1969: 432) résume et systématise de façon très claire tout le processus. D'après son exposé (que parfois nous compléterons), le mouvement de restitution entraîne d'abord les mots français qui rappellent de près le latin, des mots savants: bénévole, céleste, débile, décadence, décembre, déduction, etc. Mais la régression concerne aussi des mots de formation populaire. Pour certains, le modèle latin était encore perceptible: défendre, dé- 
sir, égal, héritier, péril, etc. Mais pour d'autres le latin ne pouvait plus être présent à l'esprit: béton, félon, hérisser, séjour. La restitution se produit encore sous l'influence de mots qui commençaient par le préfixe dis- $>$ dẹ- en français et qui va influencer des mots comme débonnaire, dévisager, débuter, etc., issus de locutions prépositionnelles avec de: de bon aire, de visage.

Le changement se produit toujours pour le préfixe præ $>$ prẹ $>$ prę $>$ prœ qui reprend un e. Par exemple dans les mots prévoir, prévôt, prédication.

Pour le préfixe latin itératif re, la restitution a été seulement partielle: réconfort, réclamer, réduire, etc., mais: recharger, redresser, redouter, etc., d'où, parfois, des divergences utiles à des fins sémantiques comme dans reformer (former de nouveau) et réformer (améliorer).

D'autre part, les flottements qui pouvaient exister depuis le Moyen Age entre é et ẹ dans des mots comme bégayer, chérir, péter, seller, téter, message, sous l'influence de bègue, cher, selle, tette, etc., et aussi entre les voyelles ę et ẹ pour des mots du type désarmer, deshonorer, etc., la première phonétique et la deuxième analogique avec défaire, dédire, etc., ces hésitations, donc, disparaissent maintenant de la langue puisqu'on a fixé normalement la prononciation en ẹ (bégayer, chérir, désarmer, etc.).

De toute façon, il faut remarquer que la prononciation $\propto$ s'est conservée dans beaucoup de mots d'usage courant, et aussi dans des mots de la langue de la campagne. Ainsi dans: chemin, chemise, cheval, cheveu, depuis, devoir, fenêtre, jeter, lever, mener, premier, tenir, venir, etc. et dans belette, semer, fenil, fenouil, etc. En tout cas, la régression n'a pas toujours été logique, comme l'indique Fouché, parce qu'on a un ẹ dans lévrier, par exemple, tandis que levrette ou levraut conservent un $\propto$.

Un nombre considérable de changements sont définitivement confirmés pendant le XVIIe siècle, comme les listes de témoignages de Thurot (1966, I: 129 et sqq.) nous permettent de l'observer. Mais parfois l'usage a été partagé et la fixation est plus tardive.

\section{2. Évolution de La Voyelle o}

Pour la voyelle postérieure, le processus de centralisation décrit est encore plus évident. Libre ou entravée, devant une consonne orale non palatale, le q se centralise davantage à partir du IVe siècle jusqu'à arriver, pendant le VIIle siècle selon Matte, à la voyelle postérieure centralisée non arrondie $\mho$, son intermédiaire entre o et $u$, comme celle qu'on entend en français canadien dans le mot bout ou en anglais dans le mot should. Ce son ne se transforme en $u$, voyelle vélaire fermée 
arrondie, qu'au XVIle siécle, quand la tension articulatoire s'est suffisamment rétablie (cf. Matte, 1982: 108). Il faut considérer que les explications de Matte ne coïncident pas avec celles de la plupart des historiens du phonétisme, qui situent le changement de ọ en u à date littéraire médiévale. Les Bourciez (1974: 113) indiquent le XIIIe siècle, Fouché (1969: 426) le XIe siècle, Straka (1956: 264-265) et La Chaussée (1989: 89) le début du XIle siècle, Zink (1989: 72) la fin du XIIe siècle.

Les affirmations de Matte s'appuient sur des témoignages divers (cf. Matte, 1982:108). D'une part, depuis l'époque mérovingienne jusqu'au XVIle siècle existe une évidente hésitation entre les graphies $o, u$ et $o u$, surtout entre $o$ et $o u$. D'autre part, la querelle des ouistes et des non-ouistes pendant le XVIe et le XVIle siècle et les explications fournies à ce sujet par les grammairiens, prouvent la réalité de cette prononciation plus ou moins intermédiaire entre $o$ et $u$. En plus, le français du Canada, qui parfois préserve des prononciations archaïques d'après la façon de parler des premiers colons, continue à prononcer $\boldsymbol{\sigma}$ centralisé, le son intermédiaire, au lieu de u. D'autre part, Juneau dans son étude Contribution à l'histoire de la prononciation française au Québec (1972: 15-24) nous montre aussi un grand nombre d'exemples de u pour o dans les textes d'Archive de la Nouvelle France. Quant à la date du changement, située approximativement vers le VIIe siècle, elle serait attestée par les exemples relevés par Pei, qui, étudiant des documents latins du nord de la France du VIIIe siècle, a trouvé des cas où apparaît, en position tonique, la graphie $u$ au lieu de o. L'auteur arrive dans son étude à la conclusion que «la fréquence du changement de o en $u$ indique que la prononciation du o à cette époque était si fermée qu'elle ressemblait plus à celle du u qu'à celle du o». (Pei, 1932: 30. Je traduis de l'anglais).

Haudricourt et Juilland, dans leur célèbre Essai pour une histoire structurale du phonétisme français, de 1970, ont expliqué le passage de o à u par le besoin du sytème de remplir la case vide créée par le u latin quand il s'est transformé en ü. (Cf. Haudricourt et Juilland, 1970: 108-120). Matte s'oppose décidément à cette explication:

Leur interprétation est basée sur l'idée que les changements phonémiques sont le résultat d'un besoin de symétrie dans le système des oppositions (...). [Matte affirme par contre] que la symétrie est une pure coïncidence du binarisme et qu'il existe beaucoup de langues dont le système des oppositions phonémiques est souvent asymétrique. (...) Ce sont les modes phonétiques qui déterminent le caractère global des oppositions (...) et la direction que prennent les changements phonétiques et phonologiques. Que o ait rempli la case de u et que u soit devenu ü est incontestable. Mais s'il s'agissait simplement de remplir une case, pourquoi o a-t-il attendu si longtemps pour devenir $u$ ? Il a hésité entre o et u pendant près de onze siècles [c'est-à-dire du VIIe au 
XVIIe siècle], alors que la case de u a été abandonnée vers la fin de l'Empire et n'a été comblée que vers la fin du mfr. Mais ce qui est encore plus surprenant, c'est qu'on puisse croire à la symétrie comme catalyseur de changement, alors que la série commencée lors de l'antériorisation de u a pris neuf à dix siècles pour s'achever -[les palatales arrondies] $\propto$ et $\propto ̣$ n'ont été formés qu'au XIIIle siècle au plus tôt. Pendant tous ces siècles c'est l'assymétrie qui a dominé. L'explication du changement $o>u$, c'est que o est devenu u seulement quand les modes phonétiques l'ont permis. (Matte, 1982: 109 n.)

Nous sommes de façon générale d'accord avec Matte dans sa critique de la pression du binarisme, mais il faut voir aussi que les données chronologiques ne sont pas définitives. Cet auteur situe le commencement de la centralisation de u au IVe siècle et le passage à ü au XVIle siècle (Matte, 1982: 102). Haudricourt et Juilland (1970: 112), d'après Meyer-Lübke et Richter, situent le changement de u en ü au Xe siècle au plus tôt. Straka (1964: 40) et La Chaussée (1989: 186 et 197), considèrent aussi l'existence d'une phase de centralisation du u latin qui commencerait au IVe siècle, mais ces auteurs situent le passage à ü au début du VIIIe siècle, date donnée aussi par Zink (1989: 129). D'autre part, on peut se demander s'il faut attendre le XVIIe siècle pour avoir les prononciations $u$ et ü. Les témoignages allégués par Matte du français canadien populaire actuel, qui, prononçant $\mho$ d'un côté et n'ayant pas toujours ü de l'autre, reflètent des tendances que le français du XVIIe siècle avait hérité de l'ancien français, pourraient être suffisamment probants. En tout cas, Matte (1982: 102) ne propose qu'une théorie et une hypothèse chronologique.

Mais il faut encore examiner les informations fournies par d'autres importants théoriciens à propos de l'évolution de cette voyelle et regarder de près les problèmes qui se sont posés pendant le XVIe et le début du XVIle siècle.

Pour Brunot, le problème de la répartition des sons o et $u$ est «une des questions les plus embrouillées de l'histoire phonétique du français au XVle siècle» (Brunot, 1967: 251). En fait, selon Thurot, l'usage a été très partagé entre les prononciations o et $u$ pendant cette période et la confusion dépasse le cadre de la position initiale, concernant aussi la tonique. (Cf. Thurot, 1966, I: 240 et sqq.).

Meigret, en 1550, dans sa Réponse aux critiques de Peletier à ce propos, affirme qu'il y a en français des mots ambigus qui n'ont ni le o ouvert de vol, mort, fort, ni le o clos de prou, dous, couvreur. Ainsi, par exemple, le son vocalique initial de douleur ne serait ni celui de dous ni celui de dol. Rouge ou Rome auraient aussi ce son intermédiaire. Etant donné qu'il n'existe pas de caractère moyen, on doit, selon lui, laisser à l'écrivain ou à l'imprimeur le choix des graphies: «o» ou «ou». 
Thurot (1966, I: 245) affirme que la fluctuation entre o et u à la tonique est peu considérable et que beaucoup des termes où elle s'avère sont d'origine non latine ou incertaine. Il cite, parmi d'autres, les mots: ventouse, chouse, penthecouste, couste, goulfe, sanglout, etc. Les rimes démontrent le sentiment d'équivalence. Ainsi, par exemple, Tabourot dit dans son Dictionnaire des rimes françoises de 1587: «Quelques-uns riment avec les mots en ouse, ostant 1'u, et disent Tholose, espose, et tout le contraire des ouystes» (voir Thurot, 1966, I: 245). Pour le o prétonique, initial ou interne, Thurot explique que le nombre des cas où l'usage a hésité a été très considérable et donne des listes d'exemples à ce propos (cf. Thurot, 1966, I: 252 et sqq.).

Rosset (1911: 75-76) résume la question en indiquant qu'au XVle siècle, moment où la langue littéraire et savante prend d'une façon plus nette conscience d'elle-même, la mode grandissante du latinisme imprègne l'écriture. Face au chaos phonétique des variantes régionales (rappelons-nous les disputes entre Meigret, lyonnais, et Peletier du Mans), on voudra de plus en plus formuler des règles d'après le modèle de régularité du latin. Alors, du concours de deux forces, l'une naturelle qui tendait à prononcer des u et l'autre savante qui, voulant régler et distribuer, tendait à retenir ou restituer des o, naît la norme de la prononciation moderne, qui s'élabore pendant le XVIe siècle et se fixe au début du XVIIe siècle.

Les effets sur le français de la réforme érasmienne, donc, doivent être observés dans le cadre général d'une tendance vers la fixation savante de la langue. Le o initial des mots latins était prononcé selon le français, avec u ou, d'après Matte, avec un timbre proche du u ( $\mho$ ). Avec la réforme, la substitution de ọ ou de ọ à $\mho$ ou déjà à u ne s'arrête pas au latin, elle concerne aussi le français.

Fouché (1969: 427-428), de nouveau, systématise de façon très claire la réalité de la distribution qu'on a réalisée en ce qui concerne la restitution savante de o ou l'adoption de $u$ en position atone initiale. Il signale que la restitution de o en français, comme c'était le cas pour e initial et aux mêmes dates, commence par les mots savants qui rappellent le latin. Ont repris un o: colere, copie, domestique, local, modérer, novembre, objet, odieux, opiner, volonté, etc. A partir de là, la régression à ọ s'étend à des mots de formation populaire: corbeau, forêt, fromage, orgueil, portrait, poteau, rosée, rossignol, soleil, etc.

Des cas d'analogie ont pu favoriser certaines régressions. Par exemple, l'analogie avec des mots populaires à radical accentué favorise l'adoption finale de ọ ou de ọ. Dans des mots comme hôtel, ôter, fossé, côté, coteau, etc., on va conserver la solution o, sous l'influence de ọt, cợt, etc. Pour les mots du type mortel, ordure, etc., grâce à l'influence analogique de mọrt, etc., le ọ se maintient.

La régression n'a été ni complète ni entièrement logique. De plus, le mouvement savant de restitution se voit contrecarré, comme on vient de voir, par la 
force de la tendance à la fermeture et ce mouvement gagne aussi, pendant une période, les couches supérieures de la société et notamment la cour.

Pour les mêmes raisons que celles que nous avons étudiées à propos du e initial, on va conserver le $u$ dans beaucoup de mots de la langue courante, très utilisés: boudin, couleur, coupable, cousin, douleur, moulin, ouvrier, poulain, tourment, vouloir, etc. Dans ces cas, l'analogie a pu aussi favoriser le maintien, à partir de mots de la même famille à radical accentué où les o toniques, étant entravés, deviennent normalement u: couler, courtois, couper, douter, rouer, tourner, etc., ont peut-être reçu l'influence de coule, cour, coupe, doute, roue, tourne, etc.

Parfois, dans des mots de même origine, les uns auront $u$ et les autres ọ: par exemple dans fourbu, fourvoyer et forfait, forfaire, des composés de fŏris.

Enfin, il faut remarquer que les restitutions qui ont commencé à se vérifier n'ont pas toujours triomphé. Par exemple, le Dictionnaire de l'Académie dans son édition de 1694 donne la double possibilité broussailles et brossailles, forme qui ne s'est pas maintenue.

\section{CONCLUSION}

Nous avons pu remarquer qu'aux XVIe et XVIle siècles un groupe social très restreint a pu changer, en partie, le cours spontané de l'évolution phonétique en ce qui concerne les voyelles initiales e et o. Evidemment, son travail ne s'arrêta pas à ces modifications-là. Pendant cette période on va construire une prononciation (cf. Rosset 1911: 366). Les grammairiens vont fixer une langue considérée comme la seule correcte à partir de plusieurs modèles: celui d'un groupe social, celui de la mode latinisante, celui de la raison et de la clarté, celui des critères encore plus subjectifs de la beauté et de l'élégance. La nouvelle prononciation se construit pour le petit monde de la cour et des classes supérieures de Paris dans un contexte où plus de $90 \%$ de la population est paysanne et analphabète. Comme l'indique Jean Delumeau, l'humanisme ne vient pas démocratiser la culture, c'est plutôt le contraire: il a accru la distance intellectuelle entre les couches supérieures et les autres, entre ceux qui ont du temps pour se former et ceux qui doivent travailler. A partir du XVIe siècle et pendant longtemps, la culture confère de véritables lettres de noblesse. (Cf. Delumeau, 1970: 256). Au cours du XVIIe siècle les grammairiens et les salons constituent la langue aristocratique du classicisme, processus qui, évidemment, opère aussi sur la prononciation pour empêcher n'importe quelle sorte de déformations et d'écarts possibles. Mais il ne faut pas oublier que la langue populaire reste intacte pendant ces deux siècles. Le fossé qui sépare la langue correcte et aristocratique et les autres langues, celles du peuple, va cependant se combler, au moins en par- 
tie, peu à peu. D'après Rosset, dès le XVIIe siècle, s'établit de plus en plus une chaîne imitative instituée à partir de contraintes socio-économiques par laquelle les bourgeois suivent la cour et les nobles et le peuple imite le bourgeois. Comme exemple, en ce qui concerne le patois parisien, les textes écrits dans cette langue «sont de plus en plus pauvres en prononciations spéciales à mesure qu'on avance dans le XVIIIe siècle; au XIXe siècle, il n'y a plus, à proprement parler, de patois parisien». (Cf. Rosset, 1911: 377).

Pour Michel Glatigny, le besoin d'établir une norme face au chaos linguistique de la France s'instaure au XVIe siècle «au fur et à mesure que le français s'étend, à partir des cercles cultivés qui gravitent autour du pouvoir» (Glatigny, 1989: 8). La norme était à construire et elle se forme pendant ce siècle de plusieurs façons: «en voulant rester au plus près de ce qu'en dit l'usage général, en le fixant dans une perspective de réforme (...), en le réduisant à ce qui est présenté comme des lois, en le limitant au nom de normes strictes» (Glatigny, 1989: 9). Petit à petit, la notion de règle s'est précisée et Estienne et Bèze, dans le dernier quart du siècle, consacrent la norme prescriptive de façon absolument explicite.

En ce qui concerne la prononciation, à partir du XVIe siècle elle n'évoluera plus librement, comme l'indiquait Brunot. Elle sera contenue et quelquefois ramenée en arrière par des influences artificielles qui sont en lutte contre les mouvements spontanés. Le poids de l'influence savante, accompagné de la multiplication textuelle générée par l'imprimerie, dans un contexte où les prescriptions doctrinales déterminent de plus en plus la façon de parler et d'écrire de certains groupes sociaux, ce poids, disais-je, faisait dire à Brunot (1967: 243), comme l'on a déjà vu, qu'il n'y a plus dès ce siècle une vraie évolution phonétique mais plutôt des transformations où les causes générales et la volonté des hommes se mélangent.

Aux dires de cet auteur, les théoriciens du XVIe siècle n'avaient pas pu, malgré leurs efforts, fixer la prononciation d'après un modèle unique. Il fallait que les temps changent pour qu'une nouvelle réalité sociale permette au travail normatif d'avoir un public. Au XVIIe siècle, l'œuvre collective des grammairiens et des gens du monde fait naître la théorie du bon usage (cf. Brunot, 1966a: 18) pour conférer le statut de seule langue au «vrai français», c'est-àdire à celui de la cour, des privilégiés et, en général, des gens cultivés.

\section{BIBLIOGRAPHIE}

BeAulieux Ch., 1967 (1 re éd. 1927). Histoire de l'orthographe française. l. Formation de l'orthographe, des origines au milieu du XVIe siècle. II. Les accents et autres signes auxiliaires, Paris, Champion. 
Bourciez E. \& J., 1974 (1 re éd.. 1889). Phonétique française. Etude historique, Paris, Klincksieck.

BRUNOT F., 1966a (1 re éd. 1909-1911). Histoire de la langue française des origines à nos jours, $t$. III, première partie. La formation de la langue classique $1600-$ 1660, Paris, A. Colin.

BRUNOT F., 1966b (1re éd. 1913-1924). Histoire de la langue française des origines à nos jours, $t$. IV., première partie. La langue classique 1660-1715, Paris, A. Colin.

BRUNOT F., 1967 (1 re éd. 1906). Histoire de la langue française des origines à nos jours, t. II. Le XVIe siècle, Paris, A. Colin.

Delumeau J., 1970. «Renaissance et discordes religieuses», dans DUBY G. (dir.). Histoire de la France, Paris, Larousse.

FouchÉ P., 1969 (1re éd. 1958). Phonétique historique du français, 3 vol., Paris, Klincksieck.

GlatiGnY M., 1989. «Norme et usage dans le français du XVIe siècle», dans SwiGGERS P. \& VAN HOECKE W., (dirs). La langue française au XVle siecle: usage, enseignement et approches descriptives, Louvain, Leuven University Press-Peeters Louvain-Paris.

HAUDRICOURT A. \& JUILLAND A., 1970 (1 re éd. 1949). Essai pour une histoire structurale du phonétisme français, The Hague-Paris, Mouton.

JUNEAU M., 1972. Contribution à l' histoire de la prononciation française au Québec, Québec, Les presses de l'Université de Laval.

La Chaussée F. de, 1989 (1re éd. 1974). Initiation à la phonétique historique de l'ancien français, Paris, Klincksieck.

MATTE E. J., 1982. Histoire des modes phonétiques du français, Genève, Droz.

PEI M., 1932. The language of the eighth century texts in Northern France, New York, Carrenza \& Co.

RENAUDET A., 1956. «Erasme et la prononciation des langues antiques», Bibliothèque d'Humanisme et Renaissance, t. 18, pp. 190-196.

ROSSET T., 1911. Les origines de la prononciation moderne étudiées au XVIle siècle d'après les remarques des grammairiens et les textes en patois de la région parisienne, Paris, A. Colin.

STRAKA G., 1956. «La dislocation linguistique de la Romania et la formation des langues romanes à la lumière de la chronologie relative des changements phonétiques», Revue de Linguistique Romane, 20, pp. 249-267.

STRAKA G., 1964. «L'évolution phonétique du latin au français sous l'effet de l'énergie et de la faiblesse articulatoires», TraLiLi, t. II, lère partie, pp. 17-98.

ThuRot Ch., 1966 (1 re éd. 1881-1884). De la prononciation française depuis le commencement du XVIe siècle, d'après les témoignages des grammairiens, 2 vol., Genève, Slaktine Reprints.

Zink G., 1989 (1re éd. 1986). Phonétique historique du français, Paris, PUF. 